\title{
Ethanol enhances collective dynamics of lipid membranes
}

\author{
Martin D. Kaye, ${ }^{1}$ Karin Schmalzl, ${ }^{2}$ Valeria Conti Nibali, ${ }^{3}$ Mounir Tarek,${ }^{4, *}$ and Maikel C. Rheinstädter ${ }^{1,5, \dagger}$ \\ ${ }^{1}$ Department of Physics and Astronomy, McMaster University, Hamilton, Ontario, L8S 4M1 Canada \\ ${ }^{2}$ Jülich Centre for Neutron Science, Forschungszentrum Jülich, Outstation at ILL, F-38042 Grenoble Cedex 9, France \\ ${ }^{3}$ Dipartimento di Fisica, Università degli Studi di Messina, I-98100 Messina, Italy \\ ${ }^{4}$ UMR 7565, Structure et Réactivité des Systèmes Moléculaires Complexes, CNRS-Nancy University, F-54506 Vandoeuvre les Nancy, France \\ ${ }^{5}$ Canadian Neutron Beam Centre, National Research Council Canada, Chalk River, Ontario, KOJ 1JO Canada
}

(Received 18 December 2010; published 25 May 2011)

\begin{abstract}
From inelastic neutron-scattering experiments and all atom molecular dynamics simulations we present evidence for a low-energy dynamical mode in the fluid phase of a 1,2-dimyristoyl-sn-glycero-3-phoshatidylcholine (DMPC) bilayer immersed in a 5\% water/ethanol solution. In addition to the well-known phonon that shows a liquidlike dispersion with energies up to $4.5 \mathrm{meV}$, we observe an additional mode at smaller energies of $0.8 \mathrm{meV}$, which shows little or no dispersion. Both modes show transverse properties and might be related to molecular motion perpendicular to the bilayer.
\end{abstract}

DOI: 10.1103/PhysRevE.83.050907

PACS number(s): 87.16.dj, 29.30.Hs, 87.10.Tf

Lipid bilayers have long been considered to be homogeneous, largely passive fluid barriers based on Singer and Nicholson's fluid model from 1972 [1]. However, it seems to be increasingly accepted that the diversity and composition of lipids play an important role in the function of biological membranes [2]. Bilayers regulate membrane elasticity, mediate protein-protein interactions [3], and are speculated to be important for transmembrane transport of small molecules [4-7].

Because of their ability to hydrogen bond with the head groups [Fig. 1(a)], short-chain alcohols, such as ethanol, are primarily located in the hydrophilic head group region [810], with bonding lifetimes of about $1 \mathrm{~ns}$ [10]. Ethanol has a number of effects on lipid bilayers: It decreases the gel-tofluid transition temperature $[8,11]$, has a weak effect on the area per lipid, and increases membrane fluidity and disorder [10]. Finally, it was found to increase membrane permeability $[10,12,13]$.

In this Rapid Communication we present evidence that ethanol modifies collective lipid-chain fluctuations. The impact of ethanol on membrane core dynamics was quantified by determining the collective short wavelength fluctuations on the 100-ps time scale from inelastic neutron scattering experiments and all atom molecular dynamics (MD) simulations. In addition to the acoustic mode that has been reported previously in phospholipid bilayers, we find evidence for a new low-energy mode. This excitation may be related to the enhanced permeability observed in the presence of ethanol molecules.

Highly oriented multilamellar membrane stacks of several thousands of chain deuterated 1,2-dimyristoyl-sn-glycero3-phoshatidylcholine (DMPC-d54) bilayers were prepared by spreading a solution of $25 \mathrm{mg} / \mathrm{ml}$ lipid in trifluoroethanol/chloroform (1:1) on 2-in. silicon wafers, followed by subsequent drying in vacuum. Twenty such wafers separated by small gaps were combined and aligned with respect to each other (total mosaicity about $0.5^{\circ}$ ). The deuterated

\footnotetext{
*Mounir.Tarek@srsmc.uhp-nancy.fr

${ }^{\dagger}$ rheinstadter@mcmaster.ca
}

samples had a total mass of about $400 \mathrm{mg}$. The stability of stacked bilayers in ethanol/water solutions with different concentrations was checked using $\mathrm{x}$-ray reflectivity. Bilayers in solutions with concentrations of more than $5 \%$ ethanol could not be stabilized for more than a couple of hours because of the well-known effect of thermal unbinding [14]. Stable bilayers in water $/ 5 \%$ ethanol could eventually be prepared by adding 2\% (weight/weight) polyethylene glycol (PEG) with a molecular weight of 20000 . PEG is known to exert an osmotic pressure on the membrane stack that stabilizes the lamellar structure [15]. PEG was reported not to participate in the DMPC bilayer and, therefore, not to have a direct effect on lipid structure and dynamics [16]. A concentration of 5\% ethanol (corresponding to a $1 \mathrm{M}$ ethanol solution) results in a structure where, on average, one ethanol molecule is attached to each lipid molecule [17]. The sandwich sample was completely immersed in about $40 \mathrm{ml}$ of a $\mathrm{D}_{2} \mathrm{O} / \mathrm{C}_{2} \mathrm{D}_{5} \mathrm{OD} / \mathrm{PEG}$ solution to ensure a defined alcohol concentration in the

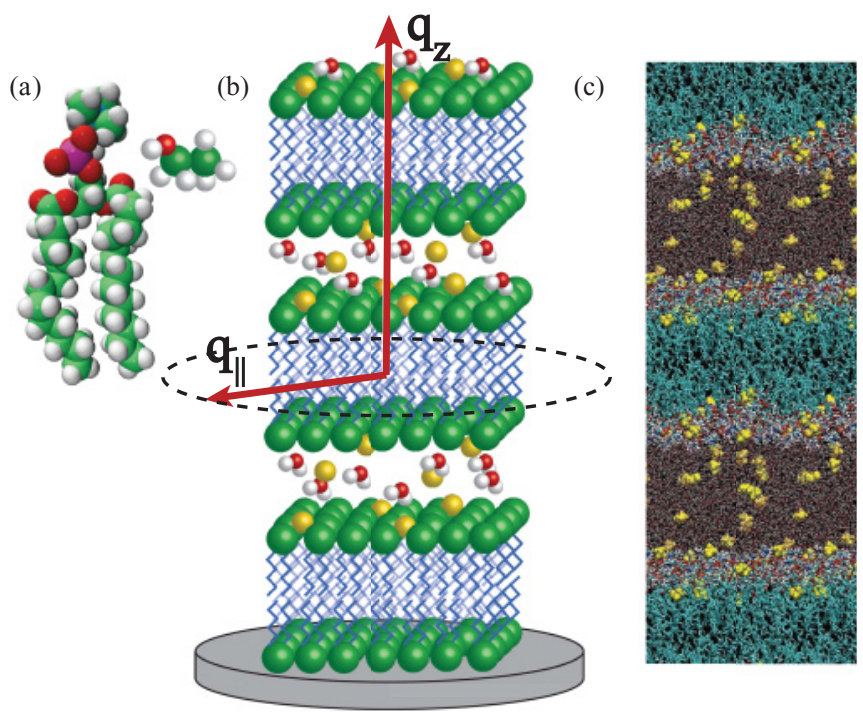

FIG. 1. (Color online) (a) Lipid (DMPC) and ethanol molecule. (b) Sketch of the supported multilamellar membrane stacks used in the neutron scattering experiment. (c) Snapshot of an all atom molecular dynamics simulation of DMPC/1M ethanol. Water molecules are depicted in red and white; ethanol molecules are depicted in yellow. 


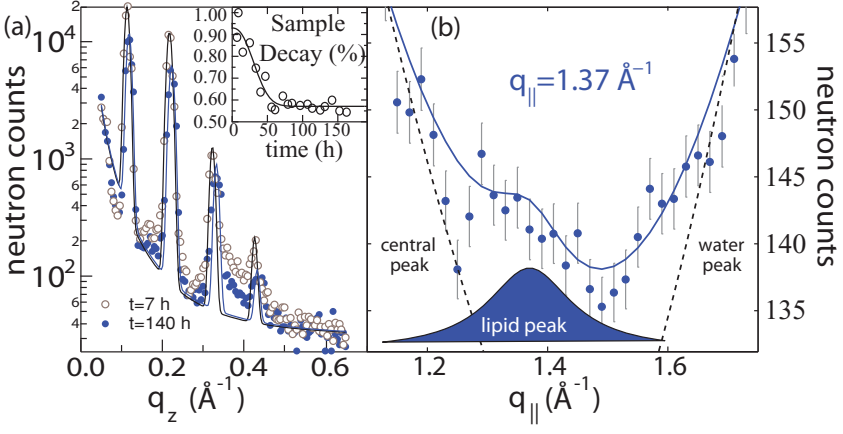

FIG. 2. (Color online) (a) Neutron reflectivity curves. (Inset) Decay of the integrated intensity of the first Bragg peak, normalized to the initial intensity. The solid line is a fit after a single exponential decay. (b) In-plane neutron diffraction of the DMPC/ethanol system. The lipid acyl chain correlation peak was observed at $q_{\|}=1.37 \AA^{-1}$.

bilayers, and mounted in a hermetically sealed aluminium container within a cryostat. Figure 1(b) shows a sketch of the supported multilamellar membrane stacks used in the neutron scattering experiment. Temperature was kept constant at $T=303 \mathrm{~K}$ during the experiment, in the fluid phase of the bilayers. ${ }^{1}$ The experiment was performed on the IN12 cold triple-axis spectrometer at the high flux reactor of the Institut Laue-Langevin, Grenoble, France. IN12 was equipped with vertically focusing monochromator and analyzer and the beam was collimated to $40^{\prime}$-monochromator-30'-sample- $30^{\prime}$ analyzer-60'-detector. Scans were done in the $\mathrm{W}$ configuration with fixed values of $k_{f}=1.75$ and $k_{f}=1.25 \AA^{-1}$, resulting in a $q$ resolution of $\Delta q=0.005 \AA^{-1}$ and energy resolutions of $\Delta \hbar \omega=200 \mu \mathrm{eV}$ and $60 \mu \mathrm{eV}$, respectively. Dynamic ranges of $\sim 4.5 \mathrm{meV}$ respectively $\sim 1 \mathrm{meV}$ were accessible using these two setups.

Figure 2(a) shows neutron reflectivity curves $\left(q_{z}\right)$ taken after the sample cell was closed and at the end of the experiment, after $t=140 \mathrm{~h}$. The lamellar spacing, $d_{z}$, was determined from the positions of the four well-developed Bragg peaks to be $d_{z}=64.5 \AA$. Note that this value is slightly lower than the value reported for DMPC under full hydration of $d_{z}=$ $66 \AA$ [18]. From MD simulations [9] ethanol was reported to decrease $d_{z}$ in DMPC/ethanol stacks. However, the decrease in $d_{z}$ must also partially be attributed to the osmotic pressure exerted by the addition of PEG to the solution. The inset in Fig. 2(a) depicts the decay of the integrated intensity of the first reflectivity Bragg peak normalized to the initial intensity with time. The solid line is a fit after a single exponential decay. Despite the use of PEG, the amount of lipids in the lamellar structure decayed to about $60 \%$ after $50 \mathrm{~h}$. Using the fit function, the counts of all scans were extrapolated to $t=0$ $\mathrm{s}$ such that all scans could be compared and combined.

In-plane diffraction of the DMPC/ethanol system $\left(q_{\|}\right)$ is shown in Fig. 2(b). The total scattering consists of a pronounced central peak at $q_{\|}=0 \AA^{-1}$ and a (heavy) water peak at about $2.0 \AA^{-1}$, the nearest-neighbor distance of the

\footnotetext{
${ }^{1}$ In multilamellar DMPC-d54 bilayers the transition from the gel into the fluid phase occurs at $T_{m}=21.5^{\circ} \mathrm{C}=294.7 \mathrm{~K}$ [19].
}

water molecules in solution. The structure factor, $S\left(q_{\|}\right)$, of the lipid tails has a maximum centered at $q_{\|}=1.37 \AA^{-1}$, corresponding to an average lipid acyl chain distance of $d=4.60 \AA$, slightly larger than in pure DMPC-d54, where $4.50 \AA$ were reported [19]. The corresponding areas per lipid can roughly be estimated from the position of the chain correlation peak [20] to be $A_{L}=2.64(9 d / 8)^{2}=70.7 \AA^{2}$ compared to $67.6 \AA^{2}$ for the pure DMPC bilayer. The area per lipid was therefore found to increase by about $4 \%$ in the presence of the ethanol molecules. We note, however, that it is not possible to compute the area per lipid directly from the interchain distance [21].

MD simulations of pure DMPC and DMPC-ethanol systems were performed at $303 \mathrm{~K}$ in the liquid crystal phase of the membrane. Each system infinitely replicated in a multilamellar array by $3 \mathrm{D}$ periodic boundary conditions contained 72 lipids, at excess hydration ( $\sim 45$ water molecules). We have considered a system at a high ethanol concentration (686 ethanol molecules partitioned in the water baths) and let it relax using constant pressure and constant temperature simulations. After $\sim 100 \mathrm{~ns}$ it equilibrated toward a final configuration where a fraction of ethanol molecules partitioned within the lipid, see Fig. 1(c) for a snapshot of the system. The DMPC and DMPC/ethanol systems were characterized by average areas per lipid of respectively 61.5 and $69.2 \AA$. These were considered in satisfactory agreement with experiment. Further simulations used to generate the scattering curves were performed at constant volume and constant temperature for duration of $4 \mathrm{~ns}$ each in order to cover the length scale corresponding to the experimental resolution. Both, the NVT and the NPT MD simulations were carried out using NAMD2 [22]. The equations of motion were integrated using a multiple time-step algorithm [23]. Short- and long-range forces were calculated every two and four time steps respectively, with a time step of $2.0 \mathrm{fs}$. Chemical bonds between hydrogen and heavy atoms were constrained to their equilibrium value. Long-range electrostatic forces were taken into account using the particle mesh Ewald (PME) approach [24]. The lipids were described by all-atom, empirical force fields proposed by Hogberg et al. [25], the water molecules by the TIP3P model [26], and the ethanol force field was taken from [27]. The connection between the MD trajectories and the inelastic neutron data is via the density correlation function. The correlation function was calculated from the MD trajectory as $I\left(q_{\|}, t\right)=\left\langle n^{*}\left(q_{\|}, 0\right) n\left(q_{\|}, t\right)\right\rangle$, where $n\left(q_{\|}, t\right)$ is the generalized density fluctuation, $n\left(q_{\|}, t\right)=1 / \sqrt{N} \sum_{\alpha} b_{\alpha} \exp \left\{i q_{\|} \cdot r_{\alpha}(t)\right\}$, with $r_{\alpha}(t)$ the position of the $\alpha$ th scatterer at time $t$ and $b_{\alpha}$ its neutron scattering length density and $N$ the number of scatterers. $S\left(q_{\|}, \hbar \omega\right)$ was calculated as a Fourier transform of $I\left(q_{\|}, t\right)$ [28]. For each wave vector selected in the range [0.4 $\AA^{-1}$ and $2 \AA^{-1}$ ], the $I\left(q_{\|}, t\right)$ s were averaged over values obtained considering a minimum of 20 randomly selected $q$ values, providing a $2 \mathrm{~d}$ powder average of the scattering. Here we focused on the lipid tail dynamics and therefore considered only contributions from the $\mathrm{C}$ atoms in the hydrocarbon chains so the experimental and simulated data can be compared directly. In addition, simulation data were convoluted with an energy resolution of $80 \mu \mathrm{eV}$, comparable to the experimental instrumental resolution. The MD data were also used to calculate the transverse dynamics of the lipid tails. The transverse current spectra, $C_{T}\left(q_{\|}, \omega\right)$, were 

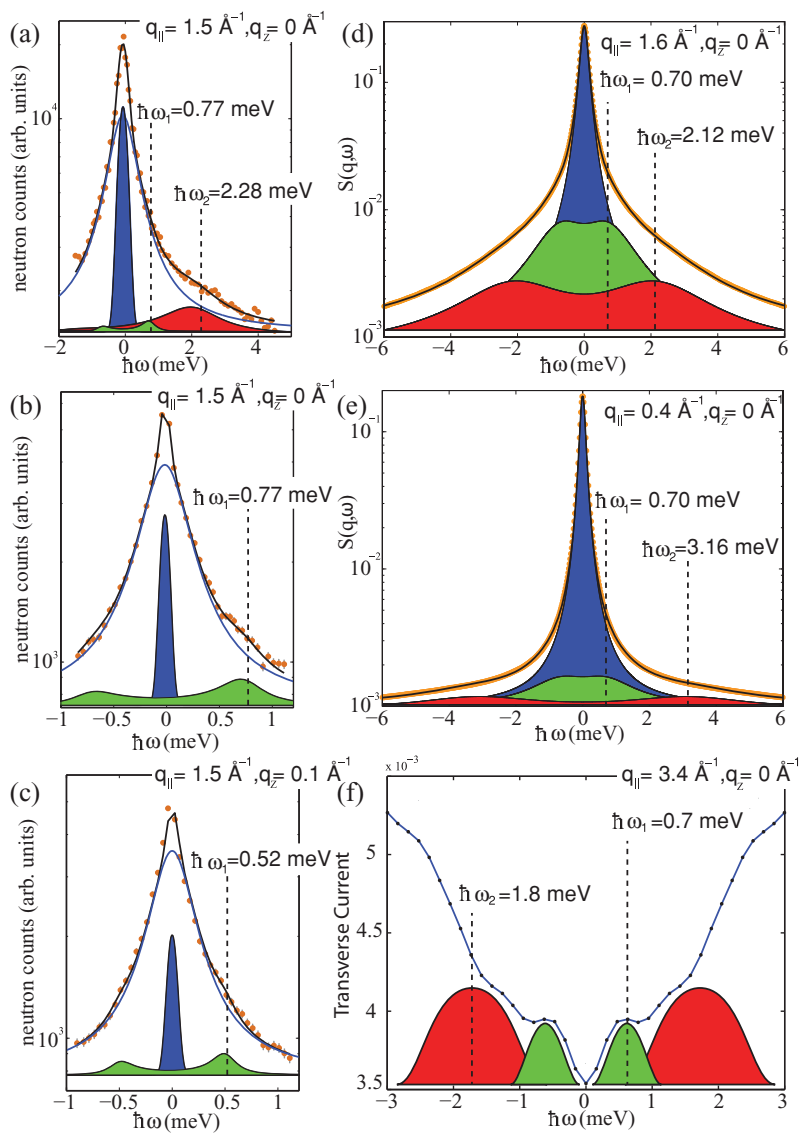

FIG. 3. (Color online) Spectra from experiment [(a), (b) and c)] and simulations [(d) and e)]. Two excitations are visible at $q_{\|}=1.5 \AA^{-1}$ in (a). Part (b) depicts the low-energy excitation at a higher energy resolution. In (c) a small $q_{z}$ component was added to the total scattering vector and an excitation was observed at $0.5 \mathrm{meV}$. Simulation data are shown for $q_{\|}=1.6 \AA^{-1}$ (d) and $q_{\|}=0.4 \AA^{-1}$ (e). (f) Calculated transverse current spectra. Two propagating modes are visible at high $q_{\|}$.

calculated following [29]. Note that these dynamics are not directly accessible experimentally [29].

Figure 3 shows the collective short wavelength dynamics of the DMPC-ethanol system from experiment and simulations. The neutron data in Figs. 3(a), 3(b), and 3(c) show a central (Gaussian) peak due to instrumental resolution and a quasielastic broadening centered at energy transfer $\hbar \omega=0 \mathrm{meV}$. This quasielastic component has contributions from coherent and incoherent scattering of the bilayers and solvent and could well be fitted by a Lorentzian peak shape. In addition, inelastic peaks were observed due to propagating collective in-plane dynamics of the deuterated membrane core. Two peaks were observed in Fig. 3(a), one at an energy of $2.28 \mathrm{meV}$ and a second one at a smaller energy of $0.77 \mathrm{meV}$. The latter is more evident in the high-resolution experiment [Fig. 3(b)]. The corresponding energies were determined by fitting damped harmonic oscillators (DHO) to the spectra. Phonon energies were determined at $q_{\|}$values of $q_{\|}=1.35$, 1.5 , and $1.65 \AA^{-1}$, around the lipid correlation peak, where the coherent scattering of the sample was found to be dominated by the coherent scattering of the lipid membrane core [19]. Note that the momentum transfer in the scans in Figs. 3(a) and 3(b)
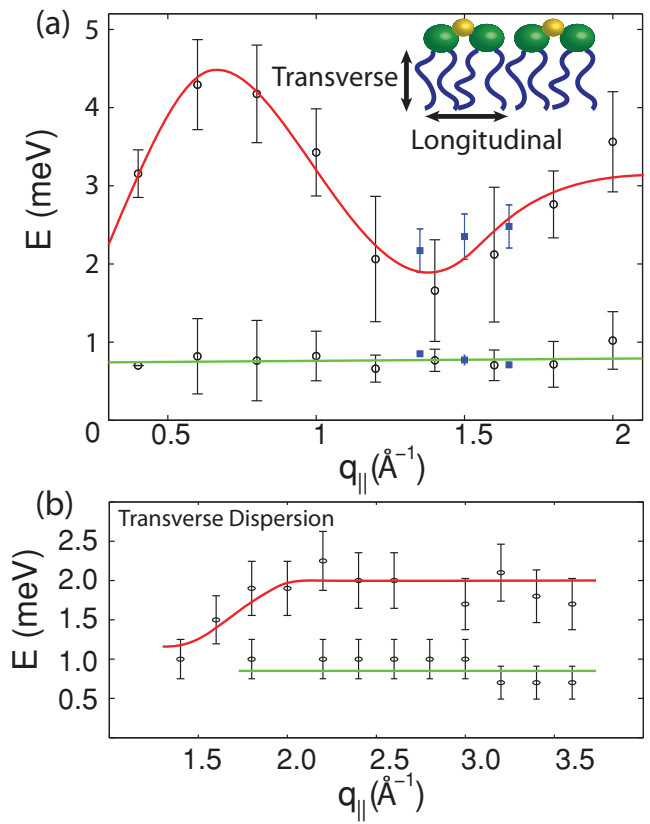

FIG. 4. (Color online) (a) Phonon energies as determined from experiment (ם) and simulation (o). While the upper branch is also found in pure lipid bilayers, the low-energy branch is attributed to the presence of the ethanol molecules in the bilayers. (b) Dispersion of the calculated transverse current spectra. Propagating transverse modes were observed for $q_{\|}$values greater than $1.5 \AA^{-1}$. Solid lines are guides for the eye.

was longitudinal and the observed modes have longitudinal properties. To determine an additional potential transverse character, additional scans have been conducted by adding a small $q_{z}$ component to the total scattering vector $\mathbf{Q}$. In the corresponding scan in Fig. 3(c), an excitation at slightly smaller energy values of $0.5 \mathrm{meV}$ was observed. Selected $S\left(q_{\|}, \hbar \omega\right)$ spectra evaluated from the MD simulations trajectories of the DMPC-ethanol system are shown in Figs. 3(d) and 3(e). The spectra were fit using the effective eigenmode model [28], which consists of a central heat mode and propagating sound modes located at $+\hbar \omega$ and $-\hbar \omega$. Two such modes were visible in the spectra and their energies were determined from the position of asymmetric Lorentzian peak shapes. Figure 3(f) shows the transverse current spectra at $q_{\|}=3.4 \AA^{-1}$. Two excitations are visible, one at $1.8 \mathrm{meV}$ and a second, low-energy, excitation at $0.7 \mathrm{meV}$. These energy values correspond to the excitation energies in the longitudinal scans within the given errors. Propagating modes could be identified in the transverse current spectra at $q_{\|}$values higher than about $1.5 \AA^{-1}$ only, corresponding to less than a nearest-neighbor lipid tail distance. The transverse dynamics are most likely relaxational at smaller $q_{\|}$values as the shear modulus is expected to be small in the fluid phase of the membranes.

The dispersion relations for longitudinal and transverse dynamics are shown in Fig. 4. Two branches are visible in the longitudinal spectra in Fig. 4(a). The dispersive mode at higher energies of about $4 \mathrm{meV}$ coincides well with the mode previously reported for pure lipid bilayers from simulations $[21,28]$ and experiments $[19,30]$. The new mode at lower 
energies of about $0.8 \mathrm{meV}$ appears to be less dispersive. Experimental and computational data for pure DMPC bilayers were analyzed in parallel and no sign of this low-energy mode was found. The latter mode was therefore attributed to the presence of the ethanol molecules in the bilayer. Two branches are also visible in the transverse current spectra in Fig. 4(b), as determined from the simulations.

An acoustic phonon branch was previously observed in lipid bilayers. This phonon has a typical shape: It shows a linear increase at small $q_{\|}$values due to long wavelength sound propagation, and a pronounced minimum at $q_{\|}$values corresponding to the nearest-neighbor distances of lipid acyl tails. Our data suggest that this mode is not altered by the presence of the ethanol molecules. The mode at $\sim 0.8 \mathrm{meV}$ not present in the pure lipid shows little or no dispersion. This conclusion is, however, mainly based on simulation data as the experimental data cover a much smaller $q_{\|}$range. The position of the low energy excitation in the experiment appears to be sensitive to the perpendicular direction as it shifts when adding a small $q_{z}$ component. Both simulations and experiment therefore point to a partial transverse, out-of-plane character of this mode. Both modes are also observed in the calculated transverse current spectra. The molecular motions associated with these modes can thus be pictured as coherent displacements of the carbon atoms in the lipid tails in the plane of the membrane, and partially also along the bilayer normal. As the collective lipid tail dynamics is related to the mobility of kink defects and small voids in the hydrophobic membrane core [6], both modes might be important for transmembrane transport. In particular the low energy mode in the presence of ethanol could be related to the enhanced transmembrane transport of small molecules. However, further investigations are needed in order to pinpoint the molecular origin of this mode.

In summary, we present the first inelastic scattering experiments to study collective lipid dynamics of supported bilayers in a water-ethanol solution complemented by all atom MD simulations. Experiment and simulation reveal an additional low-frequency collective mode associated with the acyl chain dynamics, when ethanol is embedded within the lipid bilayers. From experiment and simulations the collective hydrophobic membrane core dynamics show transverse properties, related to molecular motions perpendicular to the membrane. Whether these modes are related to transmembrane transport of small molecules will be an important question in future experiments and simulations.

We acknowledge financial support from the Natural Sciences and Engineering Research Council and National Research Council of Canada and thank the Institut Laue-Langevin for the allocation of beam time. V.C.-N. has carried out research under an HPC-EUROPA2 project (project number 228398). We are grateful to W. Treptow for help on the simulations of the initial ethanol water systems. Calculations were performed at the CINES supercomputer center (Montpellier, France).
[1] S. Singer and G. Nicolson, Science 175, 720 (1972).

[2] D. M. Engelman, Nature 438, 578 (2005).

[3] M. Rheinstädter, K. Schmalzl, K. Wood, and D. Strauch, Phys. Rev. Lett. 103, 128104 (2009).

[4] D. Huster et al., Biophys. J. 73, 855 (1997).

[5] B. Deamer and J. Bramhall, Chem. Phys. Lipids 40, 167 (1986).

[6] S. Paula et al., Biophys. J. 70, 339 (1996).

[7] G. Lahajnar et al., Biochim. Biophys. Acta 1235, 437 (1995).

[8] J. A. Barry and K. Gawrisch, Biochemistry 33, 8082 (1994).

[9] J. Chanda and S. Bandyopadhyay, Chem. Phys. Lett. 392, 249 (2004).

[10] M. Patra et al., Biophys. J. 90, 1121 (2006).

[11] S. Feller et al., Biophys. J. 82, 1396 (2002).

[12] H. Komatsu and S. Okada, Chem. Phys. Lipids 85, 67 (1997).

[13] H. V. Ly and M. L. Longo, Biophys. J. 87, 1013 (2004).

[14] M. Vogel, C. Munster, W. Fenzl, and T. Salditt, Phys. Rev. Lett. 84, 390 (2000).

[15] U. Mennicke and T. Salditt, Langmuir 18, 8172 (2002).

[16] G. Georgiev, G. Georgiev, and Z. Lalchev, Eur. Biophys. J. 35, 352 (2006).

[17] M. Jansen and A. Blume, Biophys. J. 68, 997 (1995).
[18] N. Chu, N. Kucerka, Y. Liu, S. Tristram-Nagle, and J. F. Nagle, Phys. Rev. E 71, 041904 (2005).

[19] M. Rheinstädter, C. Ollinger, G. Fragneto, F. Demmel, and T. Salditt, Phys. Rev. Lett. 93, 108107 (2004).

[20] A. Spaar and T. Salditt, Biophys. J. 85, 1576 (2003).

[21] J. S. Hub et al., Biophys. J. 93, 3156 (2007).

[22] L. Kalé et al., J. Comput. Phys. 151, 283 (1999).

[23] J. A. Izaguirre, S. Reich, and R. Skeel, J. Chem. Phys. 110, 9853 (1999).

[24] T. Darden, D. York, and L. Pedersen, J. Chem. Phys. 98, 10089 (1993).

[25] C.-J. Högberg, A. Nikitin, and A. Lyubartsev, J. Comput. Chem. 29, 2359 (2008).

[26] W. L. Jorgensen et al., J. Chem. Phys. 79, 926 (1983).

[27] M. Tarek, D. Tobias, and M. Klein, Physica A 231, 117 (1996).

[28] M. Tarek, D. J. Tobias, S. H. Chen, and M. L. Klein, Phys. Rev. Lett. 87, 238101 (2001).

[29] M. Sampoli, G. Ruocco, and F. Sette, Phys. Rev. Lett. 79, 1678 (1997).

[30] S. H. Chen et al., Phys. Rev. Lett. 86, 740 (2001). 